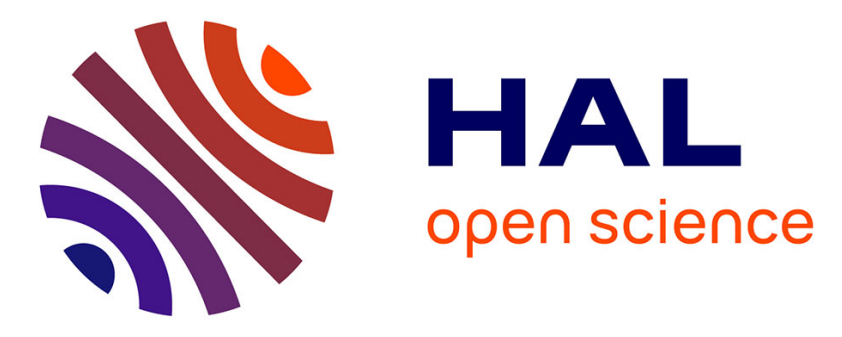

\title{
ZMP Features for Touch Driven Robot Control via Tactile Servo
}

Zhanat Kappassov, Juan Antonio Corrales Ramon, Véronique Perdereau

\section{To cite this version:}

Zhanat Kappassov, Juan Antonio Corrales Ramon, Véronique Perdereau. ZMP Features for Touch Driven Robot Control via Tactile Servo. 2016 International Symposium on Experimental Robotics, ISER 2016, International Foundation of Robotics Research, Oct 2016, Tokio, Japan. pp.234-243, 10.1007/978-3-319-50115-4_21. hal-01684799

\section{HAL Id: hal-01684799 \\ https://hal.uca.fr/hal-01684799}

Submitted on 29 Mar 2018

HAL is a multi-disciplinary open access archive for the deposit and dissemination of scientific research documents, whether they are published or not. The documents may come from teaching and research institutions in France or abroad, or from public or private research centers.
L'archive ouverte pluridisciplinaire HAL, est destinée au dépôt et à la diffusion de documents scientifiques de niveau recherche, publiés ou non, émanant des établissements d'enseignement et de recherche français ou étrangers, des laboratoires publics ou privés.

$$
\text { Copyright }
$$




\title{
ZMP features for Touch Driven Robot Control via Tactile Servo
}

\author{
Zhanat Kappassov^, Juan-Antonio Corrales Ramon ${ }^{\star \star}$, and Véronique \\ Perdereau $\star \star \star$ \\ Sorbonne Universités, UPMC Univ Paris 06, CNRS, ISIR, \\ 4 Place Jussieu, 75252 , Paris $*(\star \star \star)$ \\ Institut Pascal, BP 10448, F-63000, Clermont-Ferrand, France ${ }^{\star \star}$
}

\begin{abstract}
In most robotic applications, tactile sensors are modeled as rigid matrices of adjacent pressure sensing elements so that the geometric deformations of their surfaces are neglected. This paper proposes to define these deformations as rotational and translational compliances that are later used as new tactile features for tactile servoing tasks. In fact, a novel two-layered inverse tactile Jacobian matrix is developed in order to map errors of these features into cartesian errors required for touch-driven exploration and manipulation robotic tasks. The performance of this new tactile servoing approach is demonstrated in several real experiments with a $6 x 14$ tactile array mounted on a 7-dof robotic manipulator.
\end{abstract}

\section{Introduction}

As today robots work close with people in highly variant environment, they need to control contact with the environment to interact with unknown objects in an autonomous way. Tactile sensing provides the feedback signals required the control. The sense of touch can be given by different types of sensors, including piezo-resistive tactile sensing arrays [1]. They provide spatial contact information. By this contact information, a robot can be driven via tactile servoing [2] to perform various tasks, including autonomous exploration, object manipulation by sliding, and bimanual manipulation.

Robot motion driven by tactile feedback was implemented by Berger et al. [3] in the edge tracking problem. The authors used a 16 by 16 sensing array with compliant surface to ensure continuous feedback signal of contact pattern, and they applied a Hough transform method to estimate an orientation of the edge of an object pressed against a sensing array.

In 1991 Sikka et al. [2] introduced the tactile servoing concept. In contrast to the previous approach, the authors derived zeroth-, first-, and second- order image moments, the centroids of a contact pressure distribution as tactile features. Then the change of the feature values were mapped to the motion of

\footnotetext{
* kappassov@isir.upmc.fr, zhkappassov@nu.edu.kz

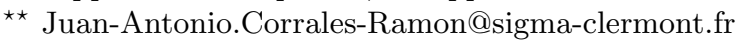

$\star \star \star$ veronique.perdereau@upmc.fr
} 
the robot end-effector in the Cartesian space. Each feature was related with one component of motion twist only.

Chen et al. [4] derived forward and inverse models of a compliant sensing surface of the tactile sensing arrays. The authors employed elasticity theory to model stress and strain distributions within the compliant layer for two types of contact: edge and point contacts on a planar surface. They showed an explicit relation between deformations at the points of contact (stresses and strains). A total force, a mean of a pressure distribution, and an orientation of an edge were derived as moments. These moments were mapped to a contact state by socalled inverse Tactile Jacobian, since it performs inverse mapping from a space of tactile features (the moments) to a space of contact states.

The main drawback of existing approaches is that the dexterity of manipulators is limited by the number of controlled dof. A motion in the six dimensional (6D) Cartesian space $\mathbb{R}^{6}$ is defined with four features. The control of moment of force is not possible when a contact area covers a sensing surface partially.

In order to overcome this disadvantage we propose new tactile features and a new task-depended two-layered inverse Tactile Jacobian. In the existing approaches [5], [2], an elastic cover of sensing arrays is used for overcoming imprecision in position control of a manipulator (robot arm). In contrast, we consider the compliance of the elastic surface of the sensor to add artificial constraints to the components of motion in $\mathbb{R}^{6}$ by introducing the new Zero-Moment-Point (ZMP) features. These features are based the on calculation of the distance between a Center of Pressure and a Center of Contact (section 2.1). This distance represents a moment of force, which in turn is related with rotational displacements through a rotational compliance of the sensing array. This information allows to control a moment of force during partial contact and, therefore, increases dexterity of the manipulators. The experimental platforms are: the seven-dof Kuka lightweight robot arm and the pneumatic (air muscle) Shadow arm with a tendon-driven robot hand.

\section{Contact Frame Control}

We consider a robot arm with a tactile sensing array on the end-effector. The arm is supposed to have contacts with other objects at the end-effector only. The robot is controlled by a set of features that are derived from a two-dimensional pressure profile referred as tactile image $I(x, y)$ acquired by a sensing array. In the following we present the algorithm to extract the proposed features, which define a contact frame, and explain how an error in the feature space is incorporated in a architecture of the position-based robot arm controller through the derived inverse tactile Jacobian matrix.

\subsection{Model and Features of a Contact on a flat and elastic surface}

We model the sensing surface as a three-dimensional spring and as an image sensor. By considering the sensor's compliance as a 3D spring, six coordinates 


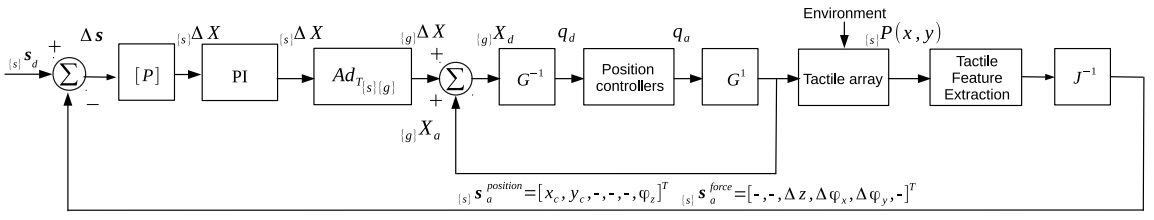

Fig. 1. Position based contact frame controller. $I(x, y)$ is a 2-D pressure distribution referred as a tactile image.

of the contact frame's origin $\boldsymbol{o}_{\boldsymbol{c}}=\left[\begin{array}{llllll}x_{c}, & y_{c}, & \Delta z, & \Delta \varphi_{x}, & \Delta \varphi_{y}, & \varphi_{z}\end{array}\right]^{T}$ can be defined in $\mathbb{R}^{6}$.

Role of compliance: The main idea of our approach is that tactile sensing arrays have compliant surface, i.e. sensors are covered with an elastic rubber. Considering the contact forces and moments, the origin of the contact frame can be defined as follows ${ }_{\{s\}} \boldsymbol{o}_{\boldsymbol{c}}=\left[-,-,{ }_{\{s\}} \Delta z,{ }_{\{s\}} \Delta \varphi_{x},{ }_{\{s\}} \Delta \varphi_{y},-\right]^{T}$, where $\{s\}$ states for the sensor frame.

Modeling as an Image sensor: The rest three components of a Cartesian vector pose, that defines the contact origin, can be found by considering the tactile sensing array as an image sensor: $\left\{\boldsymbol{s}_{\boldsymbol{\beta}} \boldsymbol{o}_{\boldsymbol{c}}=\left[x_{c}, y_{c},-,-,-, \alpha_{z}\right]^{T}\right.$.

Tactile data processing. The four features, including the center of pressure $(\mathrm{CoP})$ in $\mathrm{x}$ - and y- axes, $\mathrm{CoP}_{x}$ and $\mathrm{CoP}_{y}$, the normal force, $f$, and the orientation of an edge $\alpha_{z}$ represent the variables $x_{c}, y_{c},{ }_{\{s\}} \Delta z$, and ${ }_{\{s\}} \Delta \varphi_{z}$ of the vector defining a contact frame. As in [5], these features are given by image moments. The rest two coordinates $\Delta \varphi_{x}, \Delta \varphi_{y}$ of the origin of a contact frame are represented by the proposed features, $\boldsymbol{Z M P}$ (zero-moment pressure) feature.

We calculate the center of contact $(\mathrm{CoC})$ from the tactile image that was binarized first. Then the distance between $\mathrm{CoP}$ and $\mathrm{CoC}$ represents rotational displacements due to rotational compliance of the sensing surface

$$
\varphi_{x} \sim \mathrm{CoC}_{x}-\mathrm{CoP}_{x} ; \varphi_{y} \sim \mathrm{CoC}_{y}-\mathrm{CoP}_{y}
$$

The six features, including the proposed new features, describe each of the Cartesian coordinate of a contact frame uniquely

$$
\boldsymbol{x} \triangleq\left[C o P_{x}, C o p_{y}, f,\left(C o C_{x}-C o P_{x}\right),\left(C o C_{y}-C o P_{y}\right), \alpha_{z}\right]^{T}
$$

\subsection{Contact Frame Controller}

Our proposed controller computes an increment in position and orientation from the difference between the desired contact frame and the actual contact frame that is derived from the described tactile features.

Interaction matrix and Tactile Feature map matrix. We introduce the mapping from the error vector of tactile features to a motion twist of a robot 
end-effector. The mapping consist of a tactile feature transformation matrix and Interaction matrices; it allows the use of the same controller in different tasks that have different constraints on the Cartesian components of the end-effector motion twist

$$
\Delta \boldsymbol{x}=[\mathbf{P}] \cdot \Delta \boldsymbol{s},
$$

where $\Delta s=s_{d}-\left[\mathbf{J}^{-\mathbf{1}}\right] \cdot\left[\mathrm{CoC}_{x}, \mathrm{Co}_{y}, f, C o P_{x}, C o P_{y}, \alpha\right]^{T}$ is the feature-error vector, $\Delta \boldsymbol{x}$ is an error in the $6 \mathrm{D}$ Cartesian space, $[\mathbf{P}]$ is the interaction matrix, and $\left[\mathbf{J}^{\mathbf{- 1}}\right]$ is the tactile feature transformation matrix that maps from one set of features to another set that can include either the ZMP feature for an edge type contact or $\mathrm{CoP}$ feature for a point contact type

$$
\mathbf{J}^{-\mathbf{1}}=\left[\begin{array}{cccccc}
1 & 0 & 0 & 0 & 0 & 0 \\
0 & 1 & 0 & 0 & 0 & 0 \\
0 & 0 & 1 & 0 & 0 & 0 \\
0 & -\alpha_{1} & 0 & 0 & 1 & 0 \\
-\alpha_{2} & 0 & 0 & 1 & 0 & 0 \\
0 & 0 & 0 & 0 & 0 & 1
\end{array}\right]
$$

The variables $\alpha_{i} \in[0,1]$ : with 1 for controlling the ZMP feature and 0 for rotation around a point contact (see 3 ). The interaction matrix selects how a robot's pose is adjusted based on the feature error. Since the equations 4, 3 relate the error in feature space with an increment of an end-effector pose in the Cartesian space, a resulting mapping can be refereed as an inverse tactile Jacobian matrix $\mathbf{J}^{\mathbf{- 1}}$ [6]. Therefore, the tactile feature transformation matrix is referred as $\mathbf{J}^{\mathbf{- 1}}$.

Position based Geometric task Controller: A Cartesian position controller is implemented to servo the obtained feature error $\Delta s$. The tactile outer loop gives set-points to an internal position control loop

$$
\boldsymbol{x}_{d}=\boldsymbol{x}_{a}+\Delta \boldsymbol{x},
$$

where $\boldsymbol{x}_{d}=\boldsymbol{x}(t+1)$ is the desired pose of the end-effector, $\boldsymbol{x}_{a}=\boldsymbol{x}(t)$ is the current pose given by the Forward Kinematics $G^{1}$ of the robot, and the contact frame error is defined by the eq. (3) that is translated and rotated wrt the base (global) $\{g\}$ frame of the robot; a proportional-integral (PI) regulator is added to ensure the convergence of the tactile error. The desired joint angles $q_{d}$ are given by the Inverse Kinematics $G^{-1}$. Figure 1 represents the developed controller.

\section{Applications and Experiments}

In our experiments we used a $6 \times 14$ Weiss Robotics WTS0614 array, a Kuka lightweight arm, and a pneumatic Shadow arm with Shadow robot hand. The desired pose computation rate was set to $100 \mathrm{~Hz}$. The internal position controller of the robots operates at $1 \mathrm{kHz}$. The proposed tactile servoing controller is implemented in Robot Operating System (ROS). The Kuka arm is operated in the Cartesian space compliance mode using the default stiffness and damping parameters of $1000 \mathrm{~N} / \mathrm{m}$ and $0.7 \mathrm{Ns} / \mathrm{m}$. The tactile array (sensor) is attached either to the palm of the Shadow robot hand or at the center of the ATi Gamma force sensor mounted onto the Kuka end-effector (Fig. 2 c, d). 
The maximum sampling rate of the sensor is around $270 \mathrm{~Hz}$. A sensing performance of the WTS0614 was evaluated using the a three axes manipulator, the ATI Nano17 force sensor (observer), and a custom made intender. Fig. 2 (c) shows the sensor's responses versus the ground-truth force measurements. Fig. 2 (d) shows the results of indentations of different depths. The maximum pressure given by the force over the contact area of the intender with the diameter of $1 \mathrm{~mm} F_{\max } /\left(\pi \cdot r^{2}\right)$ is equal to $\left(2 \mathrm{~N} / 0.785 \mathrm{~m}^{2}\right) \cdot 10^{6}=2.548 \cdot 10^{6} \mathrm{~Pa}$, that is $0.25 \mathrm{~N} / \mathrm{mm}^{2}$ or $2.89 \mathrm{~N} /$ taxel. There is a significant difference in the sensitivity of the tactel 15 , which is close to the center, and tactel 1 , which is located at the boarder. These irregularities is due to the fact that the rubber at the corners is not as flexible as at the center. As it was stated in robotics literature, e.g. [7], there are significant uncertainties in the data acquisition, which makes the identified characteristics of the sensor to remain only a rough estimate. The surface of the sensor was covered with a thin, flexible and transparent plastic to protect the resistive rubber in all of the experiments, excluding the ones of rolling objects (Sec. 3.2).

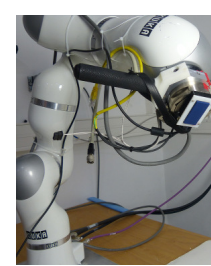

(a)

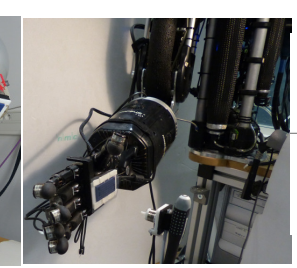

(b)

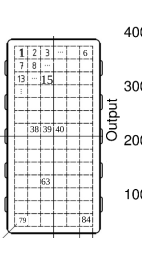

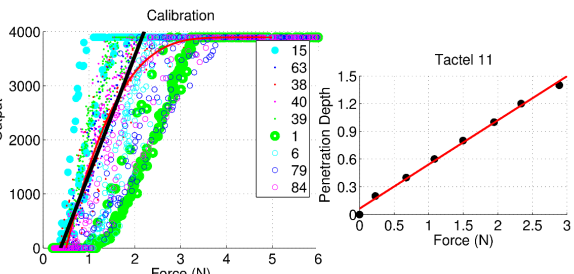

(d)

Fig. 2. The experimental setup: the Kuka arm (a) and the Shadow robot hand (b) with the WTS0614 Tactile sensor. The sensor's performance: responses of the individual sensor elements to the applied forces on each element (c) and force response to indentations of different depths (d).

\subsection{Evaluation of the tactile controller responses}

In order to verify the efficacy of the contact frame controller, two experiments are briefly outlined: simultaneous $C o C$ and force control and then the $Z M P$ feature control to illustrate the improvements of the state-of-the-art approach. The results of servoing the $\boldsymbol{C o} \boldsymbol{C}$ feature with the Shadow arm and the KUKA arm are shown in the Fig. 3 (a) and (b), respectively. The force and CoC are controlled simultaneously by setting the tactile interaction matrix to diag $(1,1,1,0,0,0)$. The response is over-dumped to prevent the overshoots that can cause the loose of the contact. The response for the air-pressure controlled arm is not as smooth as for the motor driven KUKA arm. This is due to the difference between the way of how the two arms are set in motion: in order to move the Shadow arm a pressure controller opens and clothes the valves to pump up its air-muscles 
(a soft stretchable inner rubber tubes with a braided polyester mesh sleeve). At the times of pumping the tubes, there are distortions that are neglectable in human - robot interactions, but significant in tactile servong tasks. Therefore, the following experiments are carried out using the KUKA arm only. At the times when the contact frame is moved, the contact force increases for a relatively short time period (Fig. 3). The first reason is the impact of the friction forces, a thin and slippery film was attached though. The second reason is the stick-toslip effect discussed in haptics theory, and introduced to the tactile based control approaches by Ho et al. [8]. The last reason is that the neighbor sensing elements can have different sensitivities as shown in the Fig. 2 (c). Results from control of contact force only with the Kuka arm is in accompany video [9].

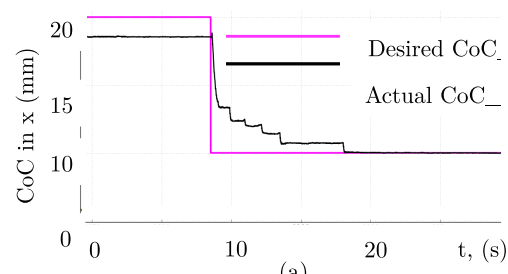

(a)

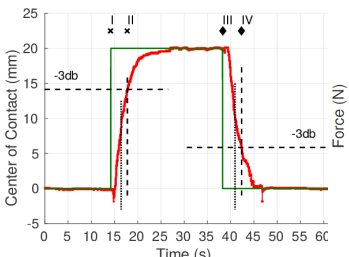

(b)

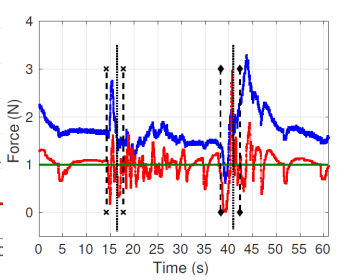

(c)

Fig. 3. Controller responses of the $C o C_{x}$ feature control with the Shadow arm (a) and the KUKA arm (b); the force responses with the KUKA arm (c). The desired, feedback, observer values are in green, red, and blue colors, respectively. The markers $\mathbf{x}$ and diamond indicate the times of new set point and the end of a transient time for the rising and falling edges, respectively.

In the robotics literature, to the best of the author's knowledge there had not been presented results of using in sliding motions and controlling the moment of force based on planar tactile sensing array. The moment of force around y-axis can be controlled using the $Z M P_{x}$ feature by setting the $\mathbf{P}$ equal to diag $(0,0,0,0,1,0)$ and the switching variable $\alpha_{1}=1$ in the $\mathbf{J}^{-\mathbf{1}}$. Fig. $4(\mathrm{a}, \mathrm{b})$ show the deformable shock absorbing foam that was used for evaluation of the $Z M P$ feature with the KUKA arm. The deformations of the sensing surface are depicted in the Fig. 4, c and d correspond to the states of the contacts shown in the Fig. 4, a and b, respectively. The Fig. ?? illustrates the ZMP feature servoing results: the proposed tactile controller can follow the desired ZMP feature given by a square-wave function (green line). The power of the proposed $Z M P$ feature is the ability to estimate a moment of force even when the edge-contact is not covering the sensing surface entirely from one to an opposite boarder of the sensor (Fig. 5, d). Limitation of the previous approaches: when an edge is not infinite, the previously proposed approaches, e.g. [5] to control the rotation around the $\mathrm{x}$ - and $\mathrm{y}$ - axes of the sensor's frame fails, since the error between the Center of Contact coordinate $C o C_{x}$ and the geometric center of the sensor 
would not converge to zero (Fig. 5, c). The video showing the evaluation of the $Z M P_{x}$ feature is available in the following link [10].

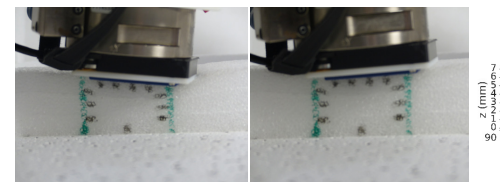

(a) (b)

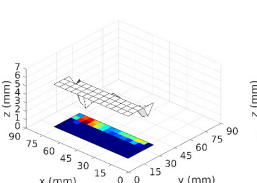

(c)

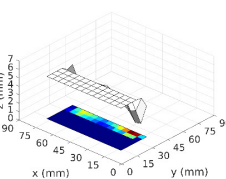

(d)

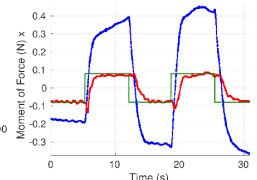

(e)

Fig. 4. Photo illustrating the control of rotational motion around y-axis based on the proposed ZMP feature representing the moment of force. A robot is applying torque in positive (a) and in negative (b) direction. The deformations of the sensing surface when the $Z M P_{x}$ is minimum (c) and maximum (d). The projected tactels with the colors represent the pressure values of each tactel: from the lowest value in blue to the highest in red. The $Z M P_{x}$ feature responses (e). The desired, feedback, observer values are in green, red, and blue colors, respectively.

\subsection{Applications and Task descriptions}

The elements of the introduced matrices can be dynamically reconfigured for different applications. In this paper we focus on three applications, including exploration of an object, manipulation by rolling and co-manipulation for keeping a tray at equilibrium.

1) An object with unknown orientation: Alignment and Exploration.

In order to explore an object, as for example metallic bar, the location of which is unknown, a robot can move its end-effector with a tactile array until the appearance of a contact (Fig. 5, a). At the presence of a contact (Fig. 5, b), the end-effector can rotate in order to align with an edge of the bar: $\alpha_{1}=0$ in the eq. (4). It is assumed that the bar lies along x-axis. When the contact area becomes large enough to compute an orientation of the appeared edge, the robot switches to control the $Z M P$ feature in order to servo an applied moment of force around y-axis: $\alpha_{1}=1$. So far, the interaction matrix is set to servo a normal force and rotation around y-axis: $\mathbf{P}=\operatorname{diag}(0,0,1,0,1,0)$.

In order to show the functionality of the reconfigurable tactile Jacobian and projection matrices, the following experiment has been carried out. The robot is not in contact and the sensing surface is not parallel with a bar at initial state (Fig. 6, a). At phase $I$ the robot follows the trajectory (Fig. 6, b) by moving through the generated posed using the KDL libraries (Fig. 6, c). At the moment of contact (Fig. 6, d), the sensor frame starts aligning with the bar by rotation linked with the $C_{o} C_{x}$ (phase $I I$ and Fig. 6,e). Then the robot switches the rotational motion around y-axis from the error in $C_{o} C_{x}$ to the error in the $Z M P_{x}$ (phase $\left.I I I\right)$. At the final phase $I V$ the robot starts the exploration driven 


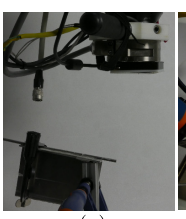

(a)

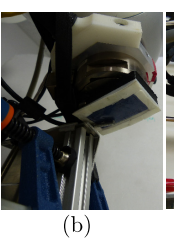

(b)

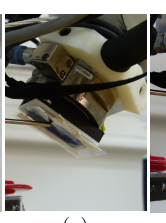

(c)

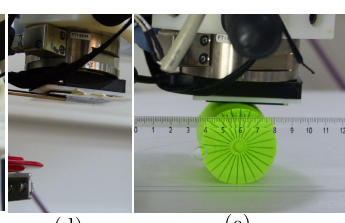

(e)

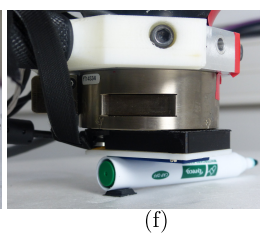

(f)

Fig. 5. Exploration of a metal bar: state "no contact" (a), "point" type contact (b), state-of-the-art approach fails to align with an edge (c), alignment with the proposed ZMP feature (d). Rolling a cylinder with the angle between two neighbor spokes equal to 15 degrees (e), rolling over rugged terrain (f)

by the error in $C o C_{x}$ but at this phase it is linked with the translational motion by reconfiguring the tactile Jacobian and projection matrices (Fig. 6, f). The yellow pixels are the recorded cloud of contact points (Fig. 6, g) at the end of exploration.

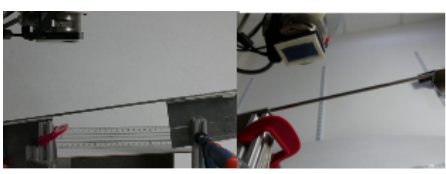

(a)

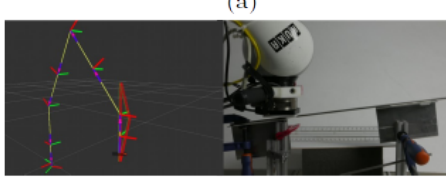

(d)

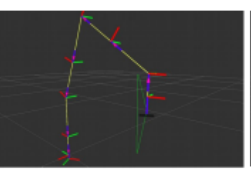

(b)

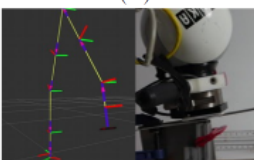

(e)

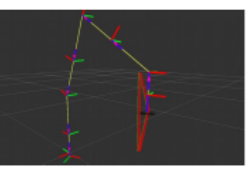

(c)

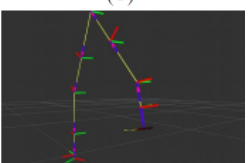

(f)

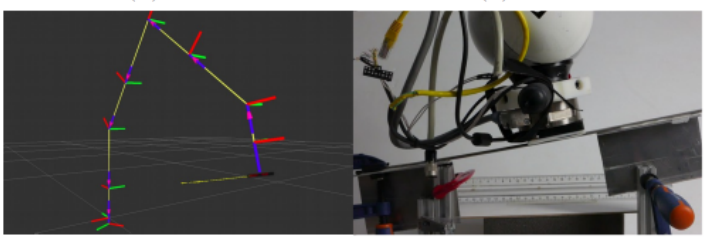

Fig. 6. Exploring an unknown shape of a metal bar: align with an edge and move along the detected edge. The initial state - a, trajectory (b) and generated poses (c) that end-effector follows until the sensor detects a contact (d), alignment with an edge (e), following the edge (f), reconstructed bar at the end of exploration (g).

2) Cylindrical object: Rolling on Rugged Terrain.

Manipulation of an object can be realized by rolling it over a sensing array (Fig. 5, e). If the object has convexities, as for example a pen cap, or the surface 
is not flat (Fig. 5, f), the rolling motion can be smoother using the proposed ZMP feature.

3) Center of Mass on a plate: manipulation to keep a balance.

When a robot is asked to hold a tray with objects on it, as for example, when there is a glass of water on the tray, which is hold by a human (co-manipulation) or by a second end-effector (bi-manual manipulation) from the opposite side, the center of mass (CoM) control is an essential task. If the CoM changes, the robot should adapt the grasping point so that the coordinates of CoM would lie on the line connecting two grasping points (Fig. 7 b, c, d); in the figure the CoM changes from $A$ to $B$, one side of the plate is attached to a static wall with a rotating bearing (Fig. 7, e), and the second side is hold by the robot with a tactile array. The target is to keep the plate in horizontal orientation, otherwise the objects on the plate will fall.

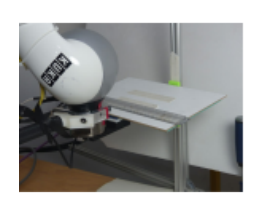

(a)

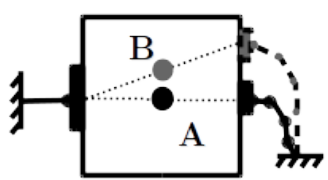

(b)

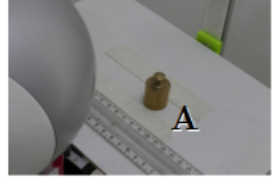

(c)

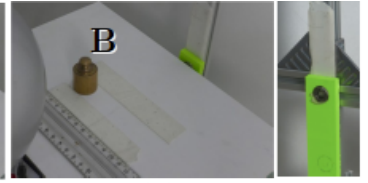

(d)

(e)

Fig. 7. Co-manipulation for keeping the equilibrium: the setup (a), Center of Mass (COM) illustration (b), COM at the center (c), COM moves (d), structure with bearing (e).

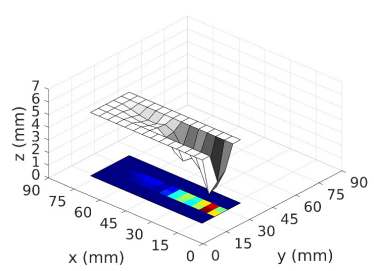

(a)

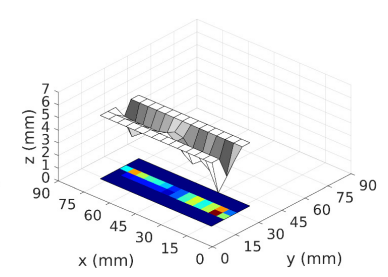

(b)

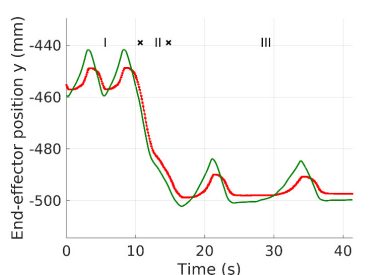

(c)

Fig. 8. Co-manipulation results: contact frame when COM moved (a), contact frame when the robot adapted with the new position of COM (b), history of the end-effector coordinate along y-axis (c). 


\section{Conclusions}

This paper introduced new tactile features, that allow a robot interacting with the environment, to control rotations around $\mathrm{x}$ - and $\mathrm{y}$-axes of its end-effector, and reconfigurable two-layered inverse Tactile Jacobian matrix, that allows to use the same controller for different tasks. The proposed new features and the inverse Tactile Jacobian matrix increase the dexterity of the robot arm-hand systems. The efficacy of the controller is validated with the experiments, in which the robot explores a shape of unknown object by sliding over an edge, manipulate cylindrical object by rolling over rugged terrain, and manipulation with the task of keeping equilibrium point of a plate.

Acknowledgments. The authors are very thankful to Vincent Hayward for discussion on tactile perception, Guillaume Walck for helping with the Shadow Hand, Vincent Padois for providing the Kuka LWR arm, and Antoine Hoarau for the help on working with the robot.

\section{References}

1. Z. Kappassov, J.-A. Corrales, and V. Perdereau, "Tactile sensing in dexterous robot hands Review," Robotics and Autonomous Systems, Jul. 2015.

2. P. Sikka, H. Zhang, and S. Sutphen, "Tactile servo: Control of touch-driven robot motion," in Experimental Robotics III. Springer, 1994, pp. 219-233.

3. A. D. Berger and P. K. Khosla, "Using tactile data for real-time feedback," The International Journal of Robotics Research, vol. 10, no. 2, pp. 88-102, 1991.

4. N. Chen, H. Zhang, and R. Rink, "Edge tracking using tactile servo," in Intelligent Robots and Systems 95.'Human Robot Interaction and Cooperative Robots', Proceedings. 1995 IEEE/RSJ International Conference on, vol. 2. IEEE, 1995, pp. 84-89.

5. Q. Li, C. Schürmann, R. Haschke, and H. Ritter, "A control framework for tactile servoing," in Robotics: Science and Systems, 2013.

6. H. Zhang and N. Chen, "Control of contact via tactile sensing," Robotics and Automation, IEEE Transactions on, vol. 16, no. 5, pp. 482-495, Oct 2000.

7. M. Prats, A. P. del Pobil, and P. J. Sanz, "Robot physical interaction through the combination of vision, tactile and force feedback," in Tracts in Advanced Robotics, ser. Springer Tracts in Advanced Robotics, B. Siciliano and O. Khatib, Eds. Springer, 2013, vol. 84, p. 177.

8. V. A. Ho, T. Nagatani, A. Noda, and S. Hirai, "What can be inferred from a tactile arrayed sensor in autonomous in-hand manipulation?" in Automation Science and Engineering (CASE), 2012 IEEE International Conference on, Aug 2012, pp. 461468.

9. Z. Kappasov, "Video showing control of force," https://www.youtube.com/watch? $\mathrm{v}=\mathrm{HK} 7 \times 51 \mathrm{U} 7 \mathrm{CiA}$, accessed April 7, 2016.

10. Z. Kappassov, "Video showing the evaluation of the proposed zmp feature," https: //www.youtube.com/watch?v=-vWIColizfo, accessed April 7, 2016.

11. Z. Kappasov, "Video showing manipulation to keep center of mass on a plate at equilibrium point," https://www.youtube.com/watch?v=TySxhsO4x9Y, accessed April 7, 2016. 